\title{
optimParallel: An R Package Providing a Parallel Version of the L-BFGS-B Optimization Method
}

by Florian Gerber and Reinhard Furrer

\begin{abstract}
The R package optimParallel provides a parallel version of the L-BFGS-B optimization method of optim(). The main function of the package is optimParallel(), which has the same usage and output as optim(). Using optimParallel() can significantly reduce the optimization time, especially when the evaluation time of the objective function is large and no analytical gradient is available. We introduce the $R$ package and illustrate its implementation, which takes advantage of the lexical scoping mechanism of $\mathrm{R}$.
\end{abstract}

\section{Introduction}

Many statistical tools involve optimization algorithms, which aim to find the minima or maxima of an objective function $f n: \mathbb{R}^{p} \rightarrow \mathbb{R}$, where $p \in \mathbb{N}$ denotes the number of parameters. Depending on the specific application different optimization algorithms may be preferred; see the book by Nash (2014), the special issue of the Journal of Statistical Software (Varadhan, 2014), and the CRAN Task View Optimization for overviews of the optimization software available for R. A widely used algorithm is the limited-memory Broyden-Fletcher-Goldfarb-Shanno algorithm with box constraints (L-BFGS-B, Byrd et al., 1996), which is available through the general-purpose optimizer optim() of the R package stats and the more recent R packages lbfgsb3 (Nash et al., 2015) and lbfgsb3c (Fidler et al., 2018). The L-BFGS-B algorithm has proven to work well in numerous applications. However, long optimization times of computationally intensive functions sometimes hinder its application; see Gerber et al. (2017) for an example of such a function from our research in spatial statistics. For this reason we present a parallel version of the optim() L-BFGS-B algorithm, denoted with optimParallel(), and explore its potential to reduce optimization times.

To illustrate the possible speed gains of a parallel L-BFGS-B implementation let $g r: \mathbb{R}^{p} \rightarrow \mathbb{R}^{p}$ denote the gradient of $f n()$. L-BFGS-B always first evaluates $f n()$ and then $g r()$ at the same parameter value and we call one such sequential evaluation one step. Note that this step should not be confused with the iteration as defined in Byrd et al. (1996) and used in the help page of opt im(), because the latter may involve several steps. Let $T_{f n}$ and $T_{g r}$ denote the evaluation times of $f n()$ and $g r()$, respectively. In the case where $g r()$ is specified in the optim() call, one step of the L-BFGS-B algorithm evaluates $f n()$ and $g r()$ sequentially, and hence, the evaluation time is little more than $T_{f n}+T_{g r}$ per step. In contrast, optimParallel() evaluates both functions in parallel, which reduces the evaluation time to little more than $\max \left\{T_{f n}, T_{g r}\right\}$ per step. In the case where no gradient is provided, optim() calculates a numeric central difference gradient approximation (CGA). For $p=1$ the CGA is defined as

$$
\widetilde{g} r(x)=(f n(x+\epsilon)-f n(x-\epsilon)) / 2 \epsilon,
$$

and hence, requires two evaluations of $f n()$. Similarly, calculating $\widetilde{g r}()$ requires $2 p$ evaluations of $f n()$ if $f n()$ has $p$ parameters. In total, optim() sequentially evaluates $f n() 1+2 p$ times per step, resulting in an elapsed time of little more than $(1+2 p) T_{f n}$ per step. Given $1+2 p$ available processor cores, optimParallel() evaluates all calls of $f n()$ in parallel, which reduces the elapsed time to little more than $T_{f n}$ per step.

\section{optimParallel() by examples}

The main function of the R package optimParallel (Gerber, 2019) is optimParallel(), which has the same usage and output as optim(), but evaluates $f n()$ and $g r()$ in parallel. For illustration, consider $10^{7}$ samples from a normal distribution with mean $\mu=5$ and standard deviation $\sigma=2$. Then, we define the following negative log-likelihood and use optim() to estimate the parameters $\mu$ and $\sigma:^{1}$

\footnotetext{
${ }^{1}$ It is obvious that simpler ways exists to estimate $\mu$ and $\sigma$. Moreover, a computationally more efficient version of negll() can be constructed based on mean() and $\operatorname{sd}()$.
} 


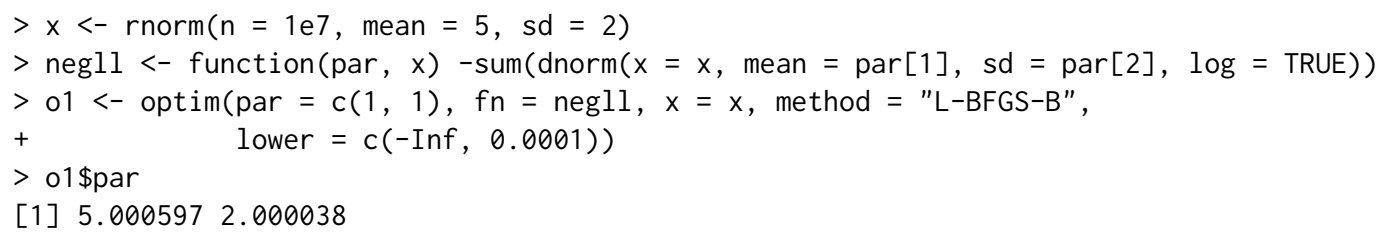

Using optimParallel(), we can do the same in parallel. The functions makeCluster(), detectCores(), and setDefaultcluster() from the R package parallel are used to set up a default cluster for the parallel execution.

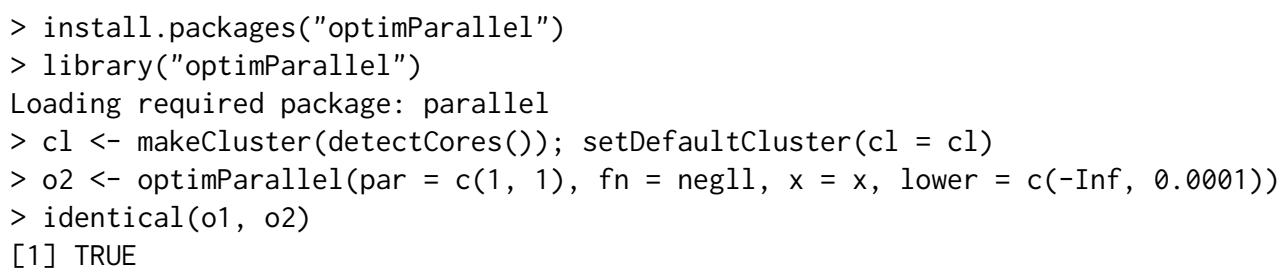

On our computer with 12 Intel Xeon E5-2640@ 2.50GHz processors one evaluation of negll() took 0.9 seconds. optim() run with 6.2 seconds per step, whereas optimParallel() run with 1.8 seconds per step. Thus, the optimization time of optimParallel() is reduced by $71 \%$ compared to that of optim(). Note that for $p=2,71 \%$ is below the maximal possible reduction of $1-1 /(2 p+1)=80 \%$ because of the overhead associated with the parallel execution and the time needed to run the L-BFGS-B algorithm, which are both not taken into account in this calculation. In general, the reduction of the optimization time is large if the parallel overhead is small relative to the execution time of $f n()$. Hence, for this example, the reduction of the optimization time approaches $80 \%$ when the evaluation time of negll() is increased, e. g., by increasing the number of data points in $\mathrm{x}$.

In addition to the arguments of optim(), optimParallel() has the argument parallel, which takes a list of arguments. For example, we can set parallel = list (loginfo $=$ TRUE) to store all evaluated parameters and the corresponding gradients.

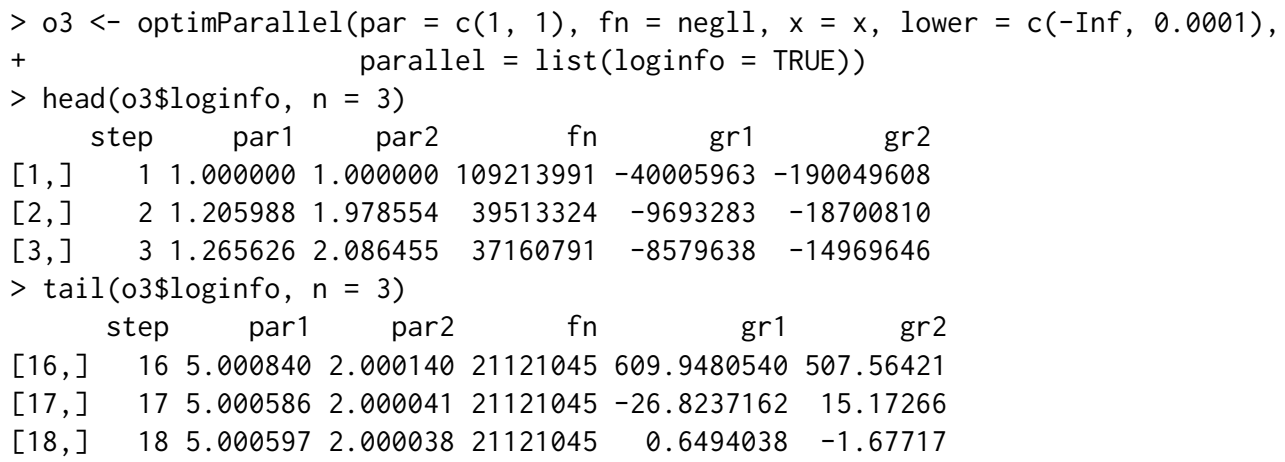

This can be used to visualize the optimization path as shown in Figure 1 and simplifies the following study, which illustrates the impact of using different (approximate) gradient specifications.

In the optimParallel() calls above the argument gr was not specified, and hence, the CGA was used. Another way of using optimParallel() is with a gradient function given to the argument gr. If the computation of the analytical gradient is tractable and does not take more time than evaluating $f n()$, this usage is preferred.

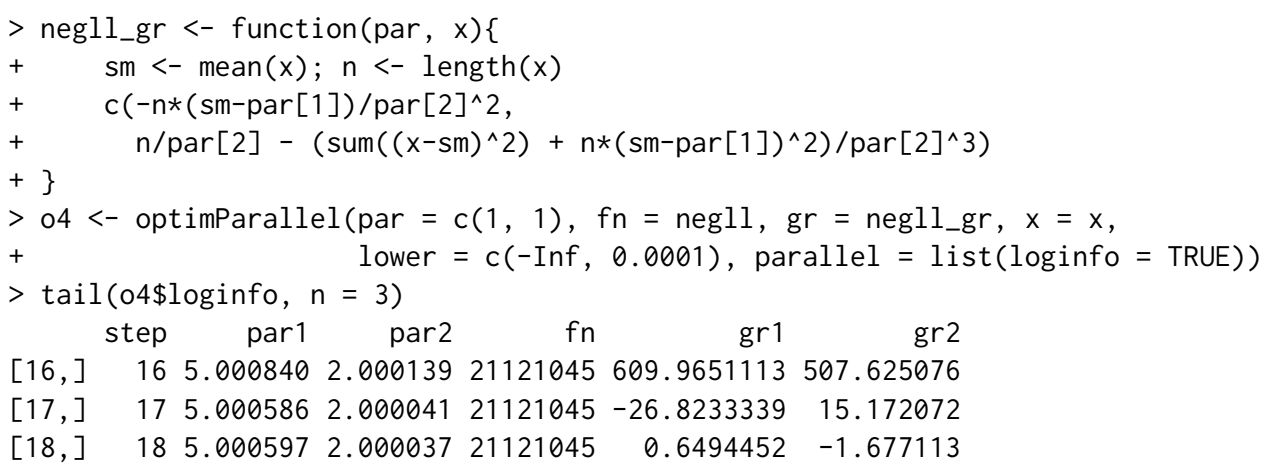

We see that the resulting optimization path is very similar to that based on the CGA ( 03 above). 


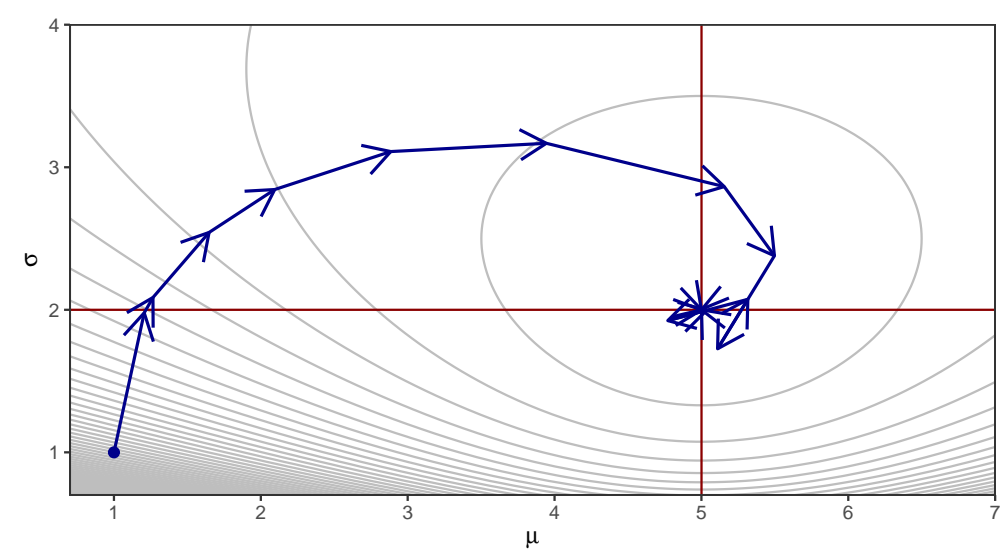

Figure 1: Visualization of the optimization path based on the log information obtained with $\operatorname{optimParallel}(\ldots$, parallel $=\operatorname{list}(\operatorname{loginfo}=$ TRUE $))$. The red lines mark the estimates $\hat{\mu}$ and $\hat{\sigma}$.

Besides the CGA and the option to explicitly specify a gradient function, optimParallel() provides a third option, namely the numeric forward difference gradient approximation (FGA) defined as $\widetilde{g r}(x)=(f n(x+\epsilon)-f n(x)) / \epsilon$ for $p=1$ and $x$ sufficiently away from the boundaries. Using the FGA, the return value of $f n(x)$ can be reused for the computation of the gradient, and hence, the number of evaluations of $f n()$ is reduced to $1+p$ per step. This can be helpful if the number of available cores is less than $1+2 p$ or insufficient memory is available to run $1+2 p$ evaluations of $f n()$ in parallel.

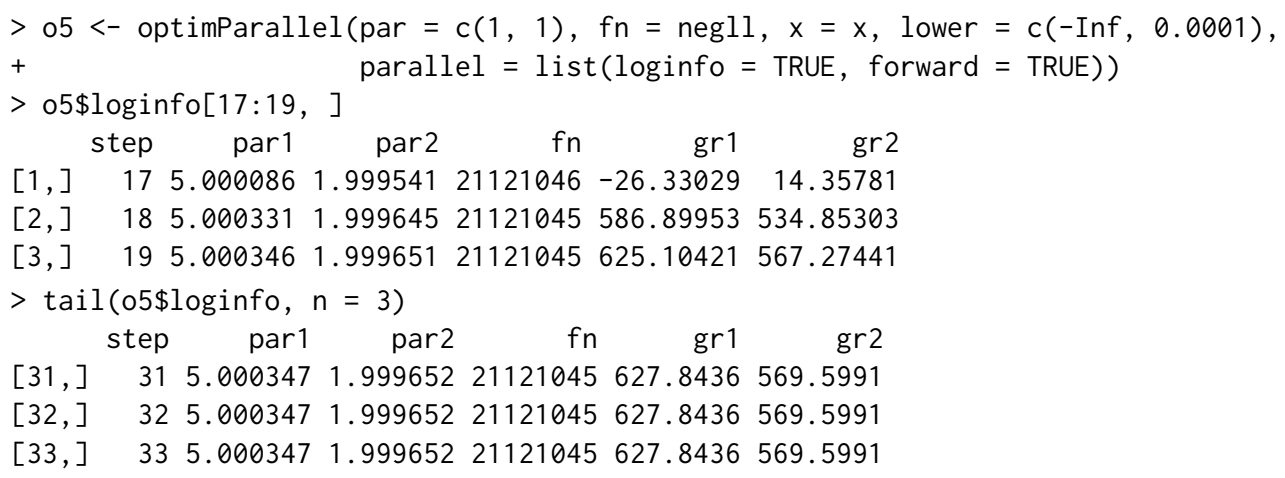

We see that the optimizer only stopped after 33 steps, whereas all previous optimization calls stopped after 18 steps. Hence, it is obvious that the choice of the gradient approximation affects the optimization. But what happened exactly?-It should be noted that the FGA is less accurate than the CGA; see, e.g., the numerical study in Nash (2014), Section 10.7. Hence, small differences in the optimization path are expected, but this does hardly explain the additional 15 steps used by the FGA based optimization. A closer inspection of the optimization path reveals that up to step 18 the path is very similar to those of the previous optimization calls and the remaining steps 19-33 only marginally change par 1 and par2. This suggests that using the FGA prevents the algorithm from stopping. One way to handle this issue is to set a less restrictive stopping criterion by increasing the value of factr.

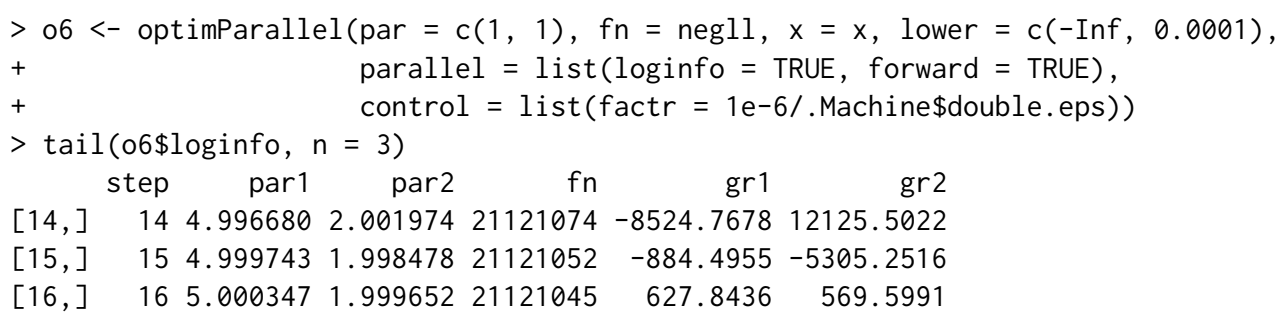

Now the resulting optimization path and the evaluation counts are similar to those resulting from the optimization using the analytic gradient ( 04 above). The take-home message of this study is that the choice of the (approximate) gradient can affect the optimization path and it may be necessary to adjust control parameters such as factr, ndeps, and parscale to obtain satisfactory results. A more detailed discussion of the use of (approximate) gradients in optimization can be found in Nash (2014), Chapter 10. 


\section{Implementation}

optimParallel() is a wrapper to optim() and enables the parallel evaluation of all function calls involved in one step of the L-BFGS-B optimization method. It is implemented in R and interfaces compiled C code only through optim(). The reuse of the stable and well-tested C code of optim() has the advantage that optimParallel() leads to the exact same results as optim(). To ensure that optimParallel() and optim() indeed return the same results optimParallel contains systematic unit tests implemented with the R package testthat (Wickham, 2017, 2011).

The basic idea of the implementation is that calling $f n()$ (or $g r())$ triggers the evaluation of both $f \mathrm{f}()$ and $\operatorname{gr}()$. Their return values are stored in a local environment. The next time $\mathrm{fn}()($ or $\operatorname{gr}())$ is called with the same parameters the results are read from the local environment without evaluating $\mathrm{fn}()$ and $\operatorname{gr}()$ again. The following R code illustrates how optimParallel() takes advantage of the lexical scoping mechanism of $R$ to store the return values of $\mathrm{fn}()$ and $\operatorname{gr}()$.

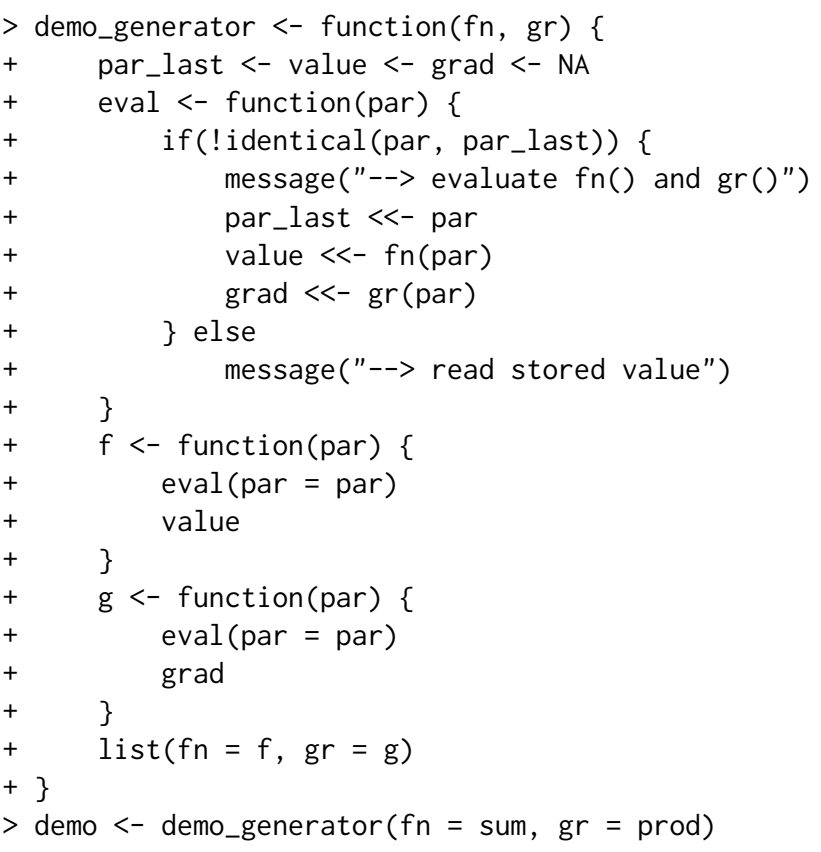

Calling demo $\$ f()$ triggers the evaluation of both $f()$ and $g r()$.

$>$ demo\$fn(1:5)

$-\rightarrow$ evaluate $f()$ and $\operatorname{gr}()$

[1] 15

The subsequent call of demo\$gr() with the same parameters returns the stored value grad without evaluating $\operatorname{gr}()$ again.

$>$ demo\$gr $(1: 5)$

$-\rightarrow$ read stored value

[1] 120

A similar construct allows optimParallel() to evaluate $f n()$ and $g r()$ at the same occasion. It is then straightforward to parallelize the evaluations of the functions using the R package parallel.

\section{Speed gains and performance test}

To illustrate the speed gains that can be achieved with optimParallel() we measure the elapsed times per step when optimizing the following test function and compare them to those of optim().

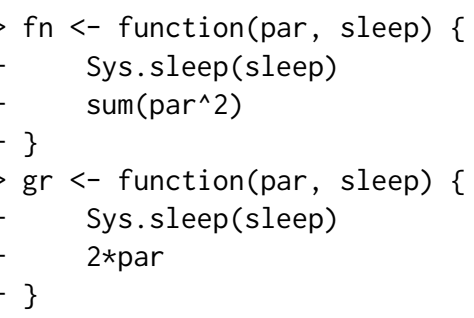




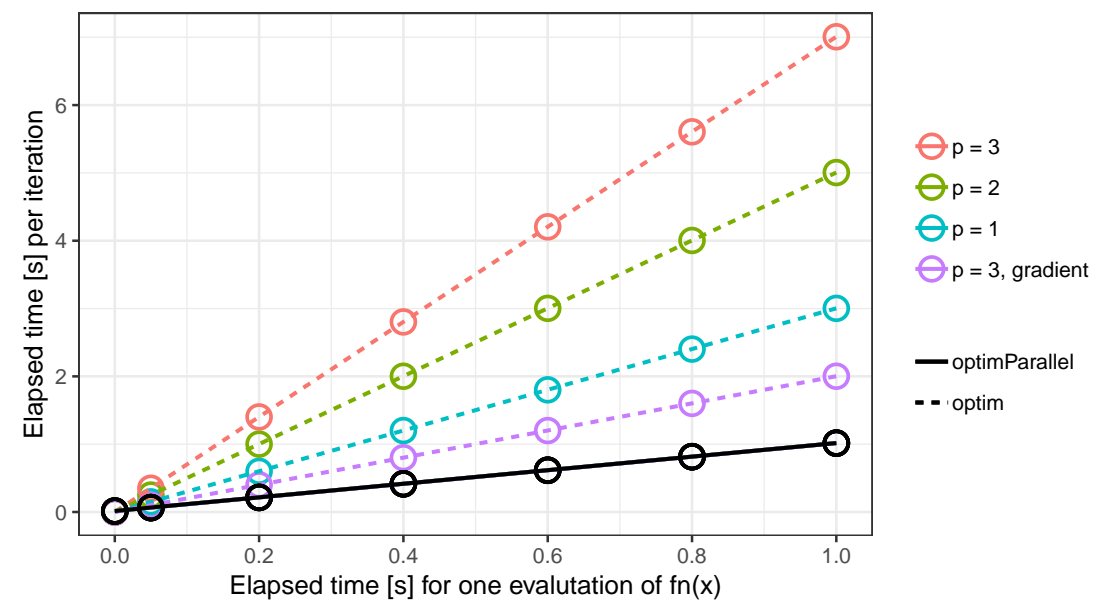

Figure 2: Benchmark experiment comparing the L-BFGS-B method from optimParallel() and optim(). Plotted are the elapsed times per step ( $y$-axis) and the evaluation time of $f n()(x$-axis) for $p=1,2$, and 3 using an approximate gradient and $p=3$ using an analytic gradient. The elapsed times of optimParallel() (solid line) are independent of $p$ and the specification of an analytic gradient.

In both functions the argument par can be a numeric vector with one or more elements and the argument sleep controls the evaluation time of the functions. We measure the elapsed time per step using all combinations of $p=1,2,3$, sleep $=0,0.05,0.2,0.4,0.6,0.8,1$ seconds with and without analytic gradient $\operatorname{gr}()$. All measurements are taken on a computer with 12 Intel Xeon E5-2640@2.50GHz processors. However, because of the experimental design a maximum of 7 processors are used in parallel. We repeat each measurement 5 times using the $\mathrm{R}$ package microbenchmark (Mersmann et al., 2018). The complete R script of the benchmark experiment is contained in optimParallel.

The results of the benchmark experiment are summarized in Figure 2. They show that for optimParallel() the elapsed time per step is only marginally larger than $T_{f n}$ (black circles in Figure 2). Conversely, the elapsed time for optim() is $T_{f n}+T_{g r}$ if a gradient function is specified (violet circles) and $(1+2 p) T_{f n}$ if no gradient function is specified (red, green, and blue circles). Moreover, optimParallel() adds a small overhead, and hence, it is only faster than optim() for $T_{f n}$ larger than 0.05 seconds.

The use of Sys.sleep() in this illustration is useful to characterize the implementation and its overhead. However, it does not represent a practical use case of optimParallel() and the speed gains might be less pronounced for other examples. One factor that reduces the speed of optimParallel() is the specification of large objects in its "..." argument. All those objects are copied to the running $\mathrm{R}$ sessions in the cluster, which increases the elapsed time. Related to that is the increased memory usage, which may slowdown the optimization when not enough memory is available.

\section{Summary}

The R package optimParallel provides a parallel version of the L-BFGS-B optimization method of optim(). After a brief theoretical illustration of the possible speed improvement based on parallel processing, we illustrate optimParallel() by examples. The examples demonstrate that one can replace optim() by optimParallel() to execute the optimization in parallel and illustrate additional features like capturing log information and using different (approximate) gradients. Moreover, we briefly sketch the basic idea of the implementation, which is based on the lexical scoping mechanism of R. Finally, a performance test shows that using optimParallel() reduces the elapsed time to optimize computationally demanding functions significantly. For functions with evaluation times of more than 0.1 seconds we measured speed gains of about factor 2 in the case where an analytic gradient was specified and about factor $1+2 p$ otherwise ( $p$ is the number of parameters). Our results suggest that using optimParallel() is most beneficial when (i) the evaluation time of $f n()$ is large (more than 0.1 seconds), (ii) no analytical gradient is available, and (iii) $p$ or more processors as well as enough memory are available. 


\section{Bibliography}

R. H. Byrd, L. Peihuang, and J. Nocedal. A limited-memory algorithm for bound-constrained optimization. Technical Report, 1996. URL https://doi .org/10.2172/204262. [p1]

M. L. Fidler, J. C. Nash, C. Zhu, R. Byrd, J. Nocedal, and J. L. Morales. Lbfgsb3c: Limited Memory BFGS Minimizer with Bounds on Parameters with Optim() 'C' Interface, 2018. URL https://CRAN. Rproject. org/package=lbfgsb3c. R package version 2018-2.13-1. [p1]

F. Gerber. optimParallel: A Parallel Version of the L-BFGS-B Method Optim(), 2019. URL https: //CRAN. Rproject. org/package=optimParallel. R package version 0.8 . [p1]

F. Gerber, K. Mösinger, and R. Furrer. Extending R packages to support 64-Bit compiled code: An illustration with spam64 and GIMMS NDVI 3 gata. Computers $\mathcal{E}$ Geosciences, 104:109-119, 2017. URL https://doi.org/10.1016/j.cageo.2016.11.015. [p1]

O. Mersmann, C. Beleites, R. Hurling, A. Friedman, and J. M. Ulrich. Microbenchmark: Accurate Timing Functions, 2018. URL https://CRAN. R-project. org/package=microbenchmark. R package version 1.4-4. [p5]

J. C. Nash. Nonlinear Parameter Optimization Using R Tools. John Wiley \& Sons, 2014. ISBN 9781118883969. URL https://doi.org/10.1002/9781118884003. [p1,3]

J. C. Nash, C. Zhu, R. Byrd, J. Nocedal, and J. L. Morales. Lbfgsb3: Limited Memory BFGS Minimizer with Bounds on Parameters, 2015. URL https://CRAN. R-project.org/package=lbfgsb3. R package version 2015-2.13. [p1]

R. Varadhan. Special volume: Numerical optimization in R: Beyond optim. Journal of Statistical Software, 6, 2014. ISSN 1548-7660. URL https://www. jstatsoft. org/issue/view/v060. [p1]

H. Wickham. Testthat: Get started with testing. The R Journal, 3:5-10, 2011. URL http://journal . Rproject.org/archive/2011-1/RJournal_2011-1_Wickham. pdf. [p4]

H. Wickham. Testthat: Unit Testing for R, 2017. URL https: //CRAN. R-project. org/package=testthat. $\mathrm{R}$ package version 2.0.0. [p 4 ]

Dr. Florian Gerber

Department of Applied Mathematics and Statistics

Colorado School of Mines, Colorado, USA

gerber@mines. edu, https: //orcid.org/0000-0001-8545-5263

Prof. Dr. Reinhard Furrer

Department of Mathematics $\mathcal{E}$ Department of Computational Science

University of Zurich, Switzerland

reinhard. furrer@math.uzh.ch, https://orcid.org/0000-0002-6319-2332 